\title{
Real-life analysis of 280 patients with surgically treated acromegaly: a single-center experience from 2008 to 2015
}

\author{
Roman Rotermund, MD, ${ }^{1}$ Marius M. Mader, MD, ${ }^{1}$ Till Burkhardt, MD, ${ }^{4}$ Jakob Matschke, MD, ${ }^{3}$ \\ Jens Aberle, MD, ${ }^{2}$ Kara Krajewski, MD, ${ }^{5}$ Jörg Flitsch, MD, ${ }^{1}$ and Amir-Hossein Rahvar, MD ${ }^{2}$
}

\begin{abstract}
1Department of Neurosurgery, ${ }^{2}|| \mid r d$ Department of Medicine, and ${ }^{3}$ Institute of Neuropathology, University Medical Center Hamburg-Eppendorf, Hamburg; ${ }^{4}$ Department of Neurosurgery, Friedrich-Ebert-Krankenhaus, Neumuenster; and ${ }^{5}$ Department of Neurosurgery, University Clinic Schleswig-Holstein, Campus Lübeck, Germany
\end{abstract}

\begin{abstract}
OBJECTIVE The purpose of this study was to analyze the clinical and biochemical outcome of consecutive patients with acromegaly after microscopic transsphenoidal surgery (MTS) at a single center over an 8-year period.

METHODS A retrospective analysis of patients with acromegaly treated via MTS between 2008 and 2015 at the authors' center was performed. The mean follow-up was 29 months (range 1-120 months). Parameters investigated included tumor size, pre- and postoperative insulin-like growth factor-I, growth hormone levels, pretreatment, perioperative complications, and clinical outcome.
\end{abstract}

RESULTS A total of 280 patients with acromegaly were treated surgically at the authors' center over the abovementioned time frame and were included in analyses. For 231 of these patients, complete follow-up data were available for evaluation. One hundred eighty-eight patients (81\%) showed remission initially according to current criteria. So far, 23 of these patients relapsed in the further course, so that on follow-up 165 patients (71\%) demonstrated full remission by surgery alone. Most patients in whom remission after surgery failed were treated with somatostatin receptor ligands and/or dopamine agonists as second-line treatment. The main postoperative complications included transient hyponatremia and diabetes insipidus (13/280; 4.6\%). CSF leakage only occurred in 2 cases (2/280; 0.7\%). No surgery-related death occurred.

CONCLUSIONS The data underline the effectiveness of MTS in acromegaly. Many patients with recurrent disease or incomplete tumor resection can be successfully managed pharmacologically.

https://thejns.org/doi/abs/10.3171/2020.3.FOCUS2061

KEYWORDS acromegaly; microscopic transsphenoidal surgery; somatostatin agonist; outcome

A CROMEGALY is a rare disease with an estimated prevalence of 30 to 70 cases per million in Europe. ${ }^{19}$ It is caused by growth hormone (GH)-producing adenomas of the pituitary gland.${ }^{14}$ The disorder was first described by Pierre Marie in 1886, as discussed by de Herder. ${ }^{5}$ Hypothalamic or ectopic causes of acromegaly are extremely rare. ${ }^{8}$ The disease's clinical manifestation in adults includes headaches, visual field defects, skin thickening, macrognathia, macroglossia, swelling of the hands and feet, deepening of the voice, and carpal tunnel syndrome. ${ }^{9}$ Further complications may include cardiovascular disease, diabetes mellitus, sleep apnea, arthropathy, and colorectal and thyroidal neoplasia. ${ }^{9}$ Generally, microscopic or endoscopic transsphenoidal surgery is performed as the first-line treatment for acromegaly. ${ }^{1}$

In addition to surgical treatment, diagnostic measures including colonoscopy, echocardiography, and thyroid ultrasound are recommended for the early detection and prevention of associated clinical complications. If clinical manifestations of acromegaly are present, serum analysis of $\mathrm{GH}$ and insulin-like growth factor-I (IGF-I) is performed. Normal IGF-I levels preclude the diagnosis of acromegaly. ${ }^{9}$ If IGF-I levels are elevated, a GH-suppression test may confirm the diagnosis of acromegaly.

Following the diagnosis of acromegaly, cranial MRI is performed. ${ }^{9}$ In most cases a macro- or microadenoma of the pituitary is visible in the cranial MRI. ${ }^{7}$ These tumors are predominantly macroadenomas (tumor size $>1$ $\mathrm{cm})$ and may invade the parasellar region. ${ }^{7,9}$ In rare cases with no visible tumor on cranial MRI but apparent clinical and biochemical diagnosis of acromegaly, an extended tumor search using PET/CT (lung/abdomen) should be performed to rule out ectopic GH secretion. ${ }^{9}$ Furthermore, serum analysis of the hypothalamic-pituitary-adrenal axis

ABBREVIATIONS DA = dopamine agonists; GH = growth hormone; IGF-I = insulin-like growth factor-I; MTS = microscopic transsphenoidal surgery; SIADH = syndrome of inappropriate antidiuretic hormone secretion; $\mathrm{SRL}=$ somatostatin receptor ligands.

SUBMITTED January 30, 2020. ACCEPTED March 3, 2020.

INCLUDE WHEN CITING DOI: 10.3171/2020.3.FOCUS2061. 
is necessary to exclude or detect hormonal insufficiencies. Visual field testing should be performed when the tumor approximates the optic chiasm.

Pharmacological treatment of acromegaly, currently considered second line after surgery, includes the use of dopamine agonists (DA) and somatostatin receptor ligands (SRL) such as octreotide or lanreotide-or as a third-line options, pasireotide and pegvisomant. ${ }^{9}$ The most common drug side effects include nausea, hyperglycemia, elevated liver enzymes, diabetes mellitus, and cholecystolithiasis. ${ }^{22}$ Ultimately, stereotactic radiotherapy or radiosurgery may be used in patients with residual tumor mass following operative treatment and an unsuccessful pharmacological approach. ${ }^{17}$ The resected tumor is analyzed histopathologically to confirm the diagnosis of GH-secreting pituitary adenoma. Recently, a revised WHO classification for the grading of pituitary tumors was released. ${ }^{11}$

Because acromegaly has a low prevalence and the diagnosis is often delayed, specialized treatment centers with surgical experience and high patient numbers are rare. In this retrospective study we performed statistical analyses on data gathered in 231 patients in whom follow-up data were available, from an initial cohort of 280 consecutive patients treated with microscopic transsphenoidal surgery (MTS) for acromegaly between January 2008 and December 2015 at our center. The goal of this study was to give a systematic overview of the clinical, biochemical, and radiological course of this rare disease in a consecutive patient cohort by using the most modern remission criteria according to the current guidelines on acromegaly by the Endocrine Society. ${ }^{9}$

\section{Methods}

Approval of the study was obtained by the local ethics committee. All patients provided informed, written consent. The study was performed in accordance with the regulations of the Declaration of Helsinki.

All consecutive patients undergoing MTS for acromegaly at our medical center between January 2008 and December 2015 were included in the study. As previously described, the general surgical technique was a transsphenoidal microscopic approach with patients in a semisitting position and with their head rotated toward the surgeon. ${ }^{12,20}$

To confirm the diagnosis of acromegaly, a standard neuropathological workup was conducted by fixing the intraoperative specimens in buffered formalin for at least 2 hours before embedding all tissue in paraffin; following standard laboratory procedures, $4-\mu \mathrm{m}$ paraffin sections were stained for $\mathrm{H} \& \mathrm{E}$ and periodic acid-Schiff reaction. Immunohistochemistry for pituitary hormones (adrenocorticotropic hormone, somatotropic hormone, prolactin, follicle-stimulating hormone, luteinizing hormone, thyroid-stimulating hormone); S100 protein; pancytokeratin (KL1 or Cam5.2); mitotic marker phosphohistone-3 (PH3); proliferation marker Ki-67 (MIB-1); and accumulation of tumor suppressor protein p53 was performed on an automated Ventana HX IHC system (Ventana-Roche Medical Systems) following the manufacturer's instructions. The classification of the tumors included somatotroph adeno- ma (either densely or sparsely granulated), mammosomatotroph adenoma, and mixed somatotroph and lactotroph adenoma. Furthermore, pharmacological and/or operative pretreatment was analyzed and taken into consideration for the assessment of clinical and biochemical outcome.

Both pre- and postoperatively, the patients' levels of spontaneous GH and IGF-I were monitored (GH: Immulite 2000 immunoassay system, Siemens; IGF-I: Liaison system, DiaSorin). GH and IGF-I were measured on the day before surgery. GH samples were drawn on the 1st and 3rd postoperative days, supplemented by collection of GH and IGF-I samples before discharge. These data were obtained in accordance with a previously published study by Rotermund et al. ${ }^{20}$ Tumor size was measured on a standard thin-sliced MRI cut (T1 weighted with and without contrast enhancement; sagittal, coronal, and axial planes) whenever available. All patients were encouraged to have a postoperative MRI session after 3-6 months to check for visible residual tumor mass, and a follow-up visit was recommended 6 months after surgery. Furthermore, the referring endocrinologists were contacted for follow-up data on the clinical course regarding remission and the values for GH and IGF-I. Remission was defined by means of all collected values according to the current criteria, with an age-related normal IGF-I and a spontaneous GH $<1 \mu \mathrm{g} / \mathrm{L}$ or $\mathrm{GH}<0.4 \mu \mathrm{g} / \mathrm{L}$ in the oral glucose tolerance test. ${ }^{9}$ Modality of the adjuvant therapy for the patients with persistent or recurrent disease was ascertained.

The data were acquired from the patients' electronic files by systematic data search. Data analyses were performed using SPSS Statistics for Windows (version 24.0; IBM Corp.). GraphPad Prism was used to draw graphs (GraphPad Software, Inc.).

\section{Results}

\section{Demographic Data}

Two hundred eighty consecutive patients underwent MTS for acromegaly at our medical center between January 2008 and December 2015.

In the overall cohort of 280 patients, 124 were male and 156 were female. The mean age was 46 years (range 6-80 years; male/female 46:46). Eighty-five patients had a microadenoma, 163 had a macroadenoma (ratio 1:2), and for the remaining 32 patients it was not possible to gather information retrospectively about the preoperative tumor size. The median tumor size (data available for 124 patients) was $1.28 \mathrm{~cm}$ (coronal), $1.36 \mathrm{~cm}$ (sagittal), and 1.28 $\mathrm{cm}$ (axial). Patient characteristics are summarized in Table 1 .

\section{History of the Present Illness}

Of the 280 patients treated surgically who later received histological confirmation of acromegaly, 267 had been referred for the treatment of clinical acromegaly. In 5 patients, the referral was for suspected nonfunctioning pituitary adenoma due to only a slight increase in IGF-I levels above the upper normal limit and without further testing. In 7 patients- 6 of whom were patients who had undergone pretreatment with DA - the leading clinical diagnosis was prolactinoma; 1 of these patients had undergone trans- 
TABLE 1. Characteristics of 280 patients with acromegaly

\begin{tabular}{lc}
\hline \multicolumn{1}{c}{ Characteristic } & Value \\
\hline Male & 124 \\
\hline Female & 156 \\
\hline Age in yrs (range) & $46(6-80)$ \\
\hline Mean age, M/F & $46: 46$ \\
\hline Adenoma size & 280 \\
\hline Macroadenomas & 163 \\
\hline Microadenomas & 85 \\
\hline No size available & 32 \\
\hline Mean measured tumor size in cm, cor/sag/ax* & $1.28 / 1.36 / 1.28$ \\
\hline Cor/sag/ax = coronal/sagittal/axial. & \\
Values are presented as number of patients unless otherwise stated. \\
* Data were available for 124 patients.
\end{tabular}

sphenoidal surgery for prolactinoma 3 years previously at a different hospital.

Ninety of 280 patients (32\%) were pretreated either surgically or pharmacologically (Table 2). Of those, 26 (9.3\% of patients overall, $28.9 \%$ of pretreated patients) presented with a relapse of a previously surgically treated adenoma. Fifteen patients (5.4\%) had been pretreated with DA, and 45 patients $(16.1 \%)$ had been pretreated with SRL.

\section{Surgery}

One patient with a giant macroadenoma underwent operation via a pterional approach and 1 patient required a retrosigmoidal approach after undergoing 5 preceding operations in order to decompress the trigeminal nerve from the tumor due to massive facial pain.

\section{Histopathological Analyses}

On neuropathological examination, 186 specimens showed isolated somatotropic immunoreactivity, whereas 94 specimens had a mammosomatotropic character. Among the most recent 133 patients who were tested for tumor granulation type, 58 specimens were sparsely granulated and 48 were densely granulated; in the other specimens there was no type of granulation documented. Fifty percent of the patients $(65 / 129$ - due to missing data 4 patients were excluded) manifested characteristics of aggressive adenomas according to the current criteria, considering that, retrospectively, no information on radiological invasiveness was available. ${ }^{10}$

\section{Laboratory Studies}

The mean preoperative IGF-I level was $734 \mu \mathrm{g} / \mathrm{L}$, the mean postoperative IGF-I level was $407 \mu \mathrm{g} / \mathrm{L}$ prior to discharge, and the mean IGF-I level at follow-up was 270 $\mu \mathrm{g} / \mathrm{L}$ (Fig. 1). The median GH levels were $48.9 \mathrm{mU} / \mathrm{L}$ preoperatively, $7.38 \mathrm{mU} / \mathrm{L}$ on postoperative day $1,9.72 \mathrm{mU} / \mathrm{L}$ on postoperative days $2-5$, and $6 \mathrm{mU} / \mathrm{L}$ before discharge (Fig. 2).

\section{Follow-Up}

Forty-nine of all patients (17.5\%) did not attend the recommended follow-up examination at our clinic after dis-
TABLE 2. Pretreatment data in 90 of 280 patients with acromegaly

\begin{tabular}{lcc}
\hline \multicolumn{1}{c}{ Finding } & No. of Patients & $\%$ \\
\hline SRL & 45 & 16.1 \\
\hline DA & 15 & 5.4 \\
\hline MTS & 9 & 3.2 \\
\hline SRL + MTS & 11 & 3.9 \\
\hline SRL + DA & 4 & 1.4 \\
\hline MTS + DA + SRL & 4 & 1.4 \\
\hline DA + MTS & 1 & 0.4 \\
\hline MTS + SRS + SSA & 1 & 0.4 \\
\hline
\end{tabular}

SRS = stereotactic radiosurgery; SSA = somatostatin agonists.

charge; follow-up was available for 231 of the 280 patients. The median time of follow-up was 29 months (1-120 months). Initial remission was achieved in $81 \%$ of the patients (188/231; microadenoma 90\% [65/73], macroadenoma 77\% [108/140]-initial tumor size was not available for all patients included in follow-up analyses). Twenty-three of those patients relapsed in the further course of followup, whereas 165 of 231 patients $(71 \%)$ stayed in remission until their last follow-up (microadenoma, 60/73 [82\%]; macroadenoma, 95/140 [68\%]; not previously pretreated microadenomas, 48/52 [92\%]; not previously pretreated macroadenomas, 73/96 [76\%]). Sixty-seven patients (29\%) either remained acromegalic despite surgery or developed a relapse.

Of those 67 patients with failed remission, 38 were then treated with SRL and 17 were treated with DA (8 of them before or in addition to SRL). One patient with an aggressive atypical adenoma was offered temozolomide after failure of pharmaco- and radiotherapy, but the individual refused. One patient refused standard pharmacotherapy and instead preferred alternative medicine options. Twenty-six patients were referred for a radiotherapy consultation. Six patients underwent a second surgery, and 2 additional patients were offered a second surgery, which they currently have not agreed to undergo.

\section{Radiotherapy}

Of the 26 patients referred for radiation therapy, followup information was available for $22(85 \%)$. Five patients refused radiotherapy and 12 patients underwent stereotactic radiotherapy, whereas the remaining patients received either Gamma Knife or CyberKnife surgery. Only 3 patients reported full remission after radiotherapy on further follow-up. No relevant side effects of radiotherapy were reported by the patients.

\section{Complications}

The most common postoperative complications were transient hyponatremia and diabetes insipidus (4.6\%). In most of these cases, hyponatremia was due to syndrome of inappropriate antidiuretic hormone secretion (SIADH), occurred mainly within the 3rd and 9th postoperative days, and was primarily treated with fluid intake restriction. CSF leakage only occurred in 2 cases, on the 3rd 


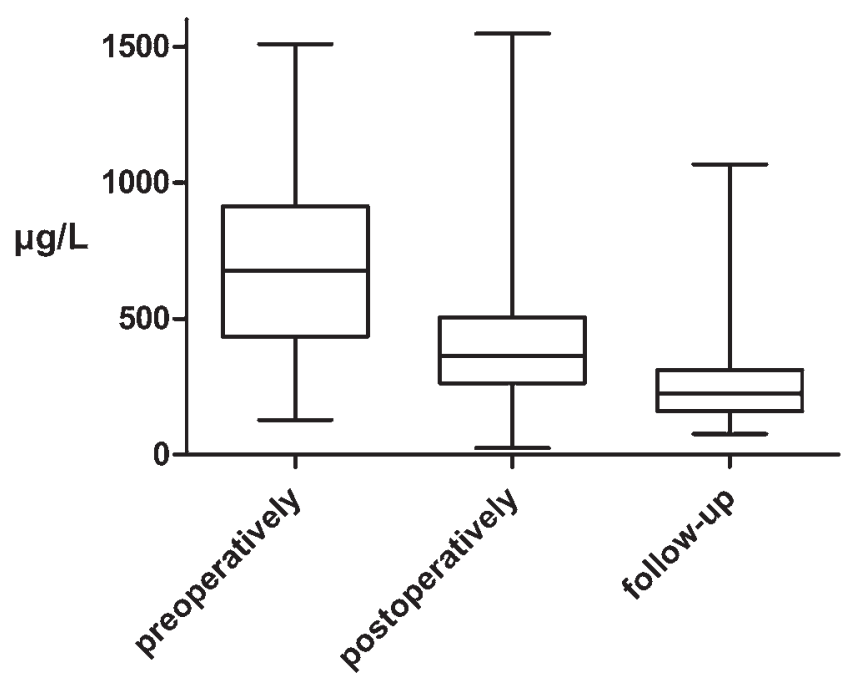

FIG. 1. Boxplot showing IGF-I levels over the course of surgical treatment.

and 7th postoperative days, respectively. Twelve patients required postoperative intensive care monitoring, in most cases due to obstructive sleep apnea. Cranial nerve defects such as hyposmia $(2 / 280 ; 0.7 \%)$ and intermittent diplopia $(2 / 280 ; 0.7 \%)$ were evaluated clinically at follow-up visits. Further perioperative complications are listed separately in Table 3.

One female patient was diagnosed with malignant melanoma 1 year after being diagnosed with acromegaly. At follow-up she reported that she felt well; the acromegaly is biochemically and radiologically in remission. After targeted chemotherapy she is also in remission for the melanoma and has further follow-ups every 6 months. Another female patient developed breast cancer 2 years after pituitary surgery and was therefore treated with adjuvant radio- and chemotherapy. Two patients died of lung cancer 2 and 4 years after diagnosis with acromegaly.

\section{Discussion}

Our data from a single expert center for a rare disease show that initial remission was achieved by MTS in $81 \%$ and "long-term" remission was achieved in $71 \%$ of cases.

In general, acromegaly has been associated with significant mortality and morbidity in the past decades. . $^{13,16,23}$ Therefore, postoperative clinical and biochemical control of acromegaly is of utmost importance to prevent further complications and to improve quality of life. Apart from the patients' direct benefits from successful surgery, the economic impact is significant as a result of the difference in costs between surgery and pharmacological treatment, because pharmacological therapy is significantly more expensive.

\section{Prognostic Factors}

Our results indicate that a larger tumor size is associated more frequently with incomplete surgical remission (90\% remission in microadenoma vs $77 \%$ in macroadenoma). Furthermore, patients receiving repeat surgeries show

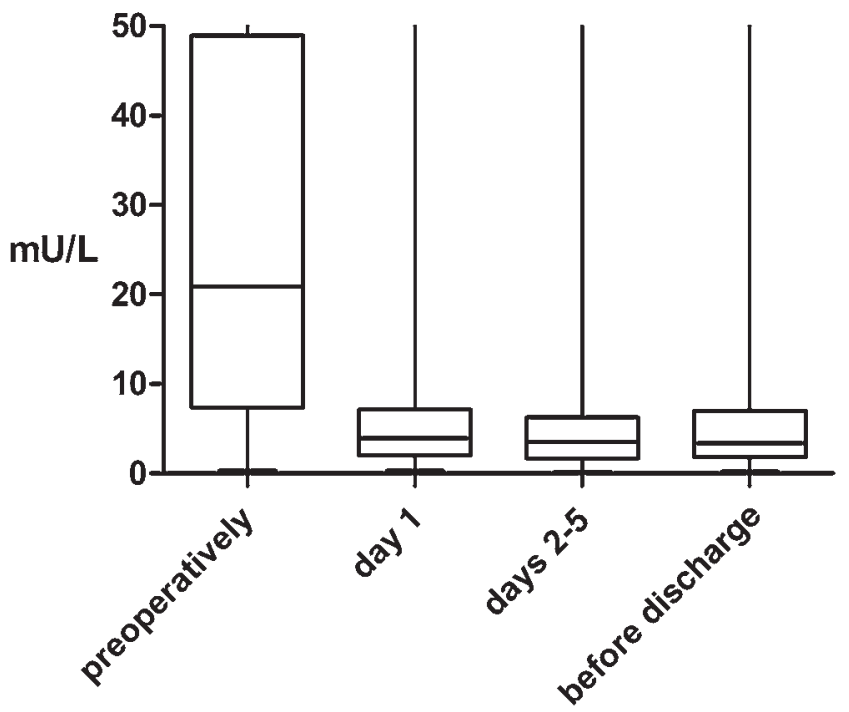

FIG. 2. Boxplot showing GH levels over the course of surgical treatment.

higher rates of incomplete remission, most likely due to the presence of scar tissue. Postoperative GH appears to have a positive predictive outcome regarding surgical and biochemical remission, as previously published by Rotermund et al. ${ }^{20}$

\section{Surgical Complications}

The most common surgical complications included postoperative hyponatremia and diabetes insipidus. These side effects were transient in nature in all patients affected. In contrast, significant reduction in tumor size as well as in GH and IGF-I levels underlines the surgical approach as the recommended first-line treatment for this disease, as suggested by the current guideline on acromegaly by the Endocrine Society. ${ }^{9}$

\section{Surgical Techniques}

Regarding the surgical approach, both microscopic and endoscopic transsphenoidal tumor resections are performed globally. Using an endoscopic approach, Babu et al. reported a full remission in $69 \%$ of patients 3 months after treatment for acromegaly via transsphenoidal surgery $(\mathrm{n}=58) .{ }^{3}$ Agam et al. reported no significant differences in outcome and complications between microscopic and endoscopic transsphenoidal surgery in 1153 consecutive operations for pituitary adenomas. ${ }^{1}$ The occurrence of the postoperative SIADH was reportedly higher in primary transsphenoidal surgery performed using an endoscopic approach compared to repeat surgery, whereas tumor size reduction was higher in primary operations..$^{18}$ In our opinion, surgical outcome and lasting remission appear to depend more on the experience of the neurosurgeon than on the specific approach. Contrary to this, Zaidi et al. proposed a quicker learning curve using an endoscopic approach compared to microscopy, although there was a significant difference in case numbers (endoscopic 100 cases vs microscopic 1800 cases). ${ }^{25}$ According to current literature, several specialized treatment centers have pub- 
TABLE 3. Perioperative complications in 280 patients with acromegaly

\begin{tabular}{|c|c|c|}
\hline Complication & Comment & $\%$ \\
\hline \multicolumn{3}{|l|}{ Intraop } \\
\hline 1 cardiopulmonary resuscitation (cardiac arrest) & $\begin{array}{l}\text { No further disadvantages, patient was still in remission at time of } \\
\text { follow-up }\end{array}$ & 0.4 \\
\hline 1 injured carotid artery; repaired immediately & $\begin{array}{l}\text { Alive; in angiographic controls no dissection/pseudoaneurysm of } \\
\text { artery was seen, \& patient is in remission }\end{array}$ & 0.4 \\
\hline \multicolumn{3}{|l|}{ Directly postop } \\
\hline 13 diabetes insipidus/SIADH & Each transient & 4.6 \\
\hline $1 \mathrm{CN}$ palsy w/ diplopia & CN III & 0.4 \\
\hline 2 CSF leakage & Op on days $3 \& 7$ & 0.7 \\
\hline 1 postop bleeding into residual tumor tissue & Conservative treatment, no permanent damage & 0.4 \\
\hline 5 epistaxis & & 1.8 \\
\hline 12 needed intensive care & Mostly due to obstructive sleep apnea & 4.3 \\
\hline \multicolumn{3}{|l|}{ At later follow-up } \\
\hline 9 hyponatremia & Each transient & 3.2 \\
\hline 4 epistaxis & & 1.4 \\
\hline Access morbidity & $\begin{array}{l}1 \text { airway obstruction, } 3 \text { sinusitis, } 4 \text { epistaxis, } 2 \text { delayed CSF leak- } \\
\text { ages ( } 2 \mathrm{w} / \text { reop) }\end{array}$ & 3.6 \\
\hline $\mathrm{CN}$ defects & $\begin{array}{l}2 \text { hyposmia, } 2 \text { intermittent double vision (CN III \& VI), } 1 \text { facial hyp- } \\
\text { esthesia (CN V, branch 2), } 1 \text { mild dysphagia since op }\end{array}$ & 2.1 \\
\hline Cardiovascular & 1 pulmonary embolism 2 mos postop, 1 myocardial infarction & 0.7 \\
\hline Others & $\begin{array}{l}1 \text { w/ sleep disturbances since op, } 1 \text { persistent libido reduction, } 1 \\
\text { amnestic dysphasia }\end{array}$ & 1.1 \\
\hline
\end{tabular}

$\mathrm{CN}=$ cranial nerve .

lished similar remission rates regarding endoscopic transsphenoidal surgery and MTS.,2,10

\section{Pharmacological Therapy}

Adjuvant therapies including SRL, DA, and GH receptor antagonists deliver a potent therapeutic option in patients with recurrent adenoma or incomplete tumor resection.

Among the patients with absent remission or relapse (29\%), approximately half were treated with SRL and/or DA. The recommendation for the course of medical treatment or repeat surgery was based on tumor size, parasellar infiltration, comorbidities, and patient preference. Publications to date have demonstrated the effects of pharmacological treatment for acromegaly in cases in which further surgical treatment is not possible because of extensive tumor growth, parasellar infiltration, or patient refusal. For these patients, e.g., Sagvand et al. showed a normalization of IGF-I up to $89 \%$ in 26 patients treated with lanreotide over the course of 24 months compared to 39 surgically cured patients. ${ }^{21}$

Switching from one pharmacological concept to another may also increase the chance for biochemical control in subgroups of patients with persistent acromegaly. According to Muhammad et al. (PAPE Study), the change from pegvisomant and a first-generation SRL to pasireotide long-acting release (PAS-LAR, $60 \mathrm{mg}$ ) led to further biochemical and clinical control of acromegaly. ${ }^{15}$ Most studies emphasize hyperglycemia, cholecystolithiasis, and elevation of liver enzymes as the most serious adverse effects of using SRL to control acromegaly. ${ }^{15,24}$ Surgical debulking appears to improve the responsiveness to SRL therapy significantly compared to pharmacological treatment alone. ${ }^{6}$

\section{Follow-Up}

Adequate postoperative care, regular blood samples, and regular follow-up examinations are vitally important to ensure disease control and to assess surgical outcome. Given that full remission is defined as a biochemical control of spontaneous $\mathrm{GH}<1 \mathrm{ng} / \mathrm{ml}$ and normal IGF-I levels, the different assays used in each individual follow-up need to be comparable. ${ }^{9}$ Our patients showed a significant reduction in $\mathrm{GH}$ levels postoperatively even on discharge $(48.9 \mathrm{mU} / \mathrm{L}$ vs $6 \mathrm{mU} / \mathrm{L})$, demonstrating the rapid success of surgery. Of interest, early postoperative GH-suppression tests appear to have a positive predictive value for longterm remission, although they are currently not recommended by the Endocrine Society.,20

\section{Study Limitations}

Our study is limited partly by the retrospective character of the analysis. Furthermore, not all patients were available for follow-up analysis, especially regarding medical treatment.

\section{Conclusions}

The adequate and timely diagnosis of acromegaly and sufficient treatment of this disease's causes are of utmost importance to reduce morbidity and mortality, improve 
quality of life, and prevent further complications. The current therapeutic options such as transsphenoidal surgery (including repeat surgery), pharmacological treatment, and radiotherapy allow for the normalization of GH levels and IGF-I levels in most cases of acromegaly. Most surgical publications in the last years have dealt with the endoscopic surgical method, and so the microscopic approach has been underrepresented. Our large cohort of patients with acromegaly who underwent MTS demonstrates the value of the microscopic technique, especially with regard to low complication rates.

\section{References}

1. Agam MS, Wedemeyer MA, Wrobel B, Weiss MH, Carmichael JD, Zada G: Complications associated with microscopic and endoscopic transsphenoidal pituitary surgery: experience of 1153 consecutive cases treated at a single tertiary care pituitary center. J Neurosurg 130:1576-1583, 2019

2. Anik I, Cabuk B, Gokbel A, Selek A, Cetinarslan B, Anik Y, et al: Endoscopic transsphenoidal approach for acromegaly with remission rates in 401 patients: 2010 consensus criteria. World Neurosurg 108:278-290, 2017

3. Babu H, Ortega A, Nuno M, Dehghan A, Schweitzer A, Bonert HV, et al: Long-term endocrine outcomes following endoscopic endonasal transsphenoidal surgery for acromegaly and associated prognostic factors. Neurosurgery 81:357-366, 2017

4. Chen CJ, Ironside N, Pomeraniec IJ, Chivukula S, Buell TJ, Ding D, et al: Microsurgical versus endoscopic transsphenoidal resection for acromegaly: a systematic review of outcomes and complications. Acta Neurochir (Wien) 159:2193-2207, 2017

5. de Herder WW: The history of acromegaly. Neuroendocrinology 103:7-17, 2016

6. Fahlbusch R, Kleinberg D, Biller B, Bonert V, Buchfelder M, Cappabianca P, et al: Surgical debulking of pituitary adenomas improves responsiveness to octreotide lar in the treatment of acromegaly. Pituitary 20:668-675, 2017

7. Famini P, Maya MM, Melmed S: Pituitary magnetic resonance imaging for sellar and parasellar masses: ten-year experience in 2598 patients. J Clin Endocrinol Metab 96:1633-1641, 2011

8. Ghazi AA, Amirbaigloo A, Dezfooli AA, Saadat N, Ghazi S, Pourafkari M, et al: Ectopic acromegaly due to growth hormone releasing hormone. Endocrine 43:293-302, 2013

9. Katznelson L, Laws ER Jr, Melmed S, Molitch ME, Murad MH, Utz A, et al: Acromegaly: an endocrine society clinical practice guideline. J Clin Endocrinol Metab 99:3933-3951, 2014

10. Lenzi J, Lapadula G, D'amico T, Delfinis CP, Iuorio R, Caporlingua F, et al: Evaluation of trans-sphenoidal surgery in pituitary GH-secreting micro- and macroadenomas: a comparison between microsurgical and endoscopic approach. J Neurosurg Sci 59:11-18, 2015

11. Lopes MBS: The 2017 World Health Organization classification of tumors of the pituitary gland: a summary. Acta Neuropathol 134:521-535, 2017

12. Ludecke DK, Abe T: Transsphenoidal microsurgery for newly diagnosed acromegaly: a personal view after more than 1,000 operations. Neuroendocrinology 83:230-239, 2006

13. Maione L, Brue T, Beckers A, Delemer B, Petrossians P, Borson-Chazot F, et al: Changes in the management and comorbidities of acromegaly over three decades: the French Acromegaly Registry. Eur J Endocrinol 176:645-655, 2017

14. Melmed S: Medical progress: acromegaly. N Engl J Med 355:2558-2573, 2006

15. Muhammad A, van der Lely AJ, Delhanty PJD, Dallenga
AHG, Haitsma IK, Janssen JAMJL, et al: Efficacy and safety of switching to pasireotide in patients with acromegaly controlled with pegvisomant and first-generation somatostatin analogues (PAPE study). J Clin Endocrinol Metab 103:586-595, 2018

16. Pivonello R, Auriemma RS, Grasso LF, Pivonello C, Simeoli C, Patalano R, et al: Complications of acromegaly: cardiovascular, respiratory and metabolic comorbidities. Pituitary 20:46-62, 2017

17. Pomeraniec IJ, Kano H, Xu Z, Nguyen B, Siddiqui ZA, Silva $D$, et al: Early versus late Gamma Knife radiosurgery following transsphenoidal surgery for nonfunctioning pituitary macroadenomas: a multicenter matched-cohort study. J Neurosurg 129:648-657, 2018

18. Przybylowski CJ, Dallapiazza RF, Williams BJ, Pomeraniec IJ, Xu Z, Payne SC, et al: Primary versus revision transsphenoidal resection for nonfunctioning pituitary macroadenomas: matched cohort study. J Neurosurg 126:889-896, 2017

19. Ribeiro-Oliveira A Jr, Barkan A: The changing face of acromegaly - advances in diagnosis and treatment. Nat Rev Endocrinol 8:605-611, 2012

20. Rotermund R, Burkhardt T, Rohani Z, Jung R, Aberle J, Flitsch J: Value of early postoperative random growth hormone levels and nadir growth hormone levels after oral glucose tolerance testing in acromegaly. Growth Horm IGF Res 41:64-70, 2018

21. Sagvand BT, Khairi S, Haghshenas A, Swearingen B, Tritos NA, Miller KK, et al: Monotherapy with lanreotide depot for acromegaly: long-term clinical experience in a pituitary center. Pituitary 19:437-447, 2016

22. Schmid HA, Brue T, Colao A, Gadelha MR, Shimon I, Kapur K, et al: Effect of pasireotide on glucose- and growth hormone-related biomarkers in patients with inadequately controlled acromegaly. Endocrine 53:210-219, 2016

23. Schöfl C, Petroff D, Tönjes A, Grussendorf M, Droste M, Stalla G, et al: Incidence of myocardial infarction and stroke in acromegaly patients: results from the German Acromegaly Registry. Pituitary 20:635-642, 2017

24. Tahara S, Murakami M, Kaneko T, Shimatsu A: Efficacy and safety of long-acting pasireotide in Japanese patients with acromegaly or pituitary gigantism: results from a multicenter, open-label, randomized, phase 2 study. Endocr J 64:735747, 2017

25. Zaidi HA, Awad AW, Bohl MA, Chapple K, Knecht L, Jahnke $\mathrm{H}$, et al: Comparison of outcomes between a less experienced surgeon using a fully endoscopic technique and a very experienced surgeon using a microscopic transsphenoidal technique for pituitary adenoma. J Neurosurg 124:596-604, 2016

\section{Disclosures}

This research used a database that was financially supported by an unrestricted grant from Novartis Pharma.

\section{Author Contributions}

Conception and design: Flitsch. Acquisition of data: Rotermund, Burkhardt, Matschke. Analysis and interpretation of data: Rahvar, Rotermund, Mader, Krajewski. Drafting the article: Rahvar, Rotermund. Critically revising the article: Mader, Krajewski, Flitsch. Reviewed submitted version of manuscript: all authors. Approved the final version of the manuscript on behalf of all authors: Rahvar. Administrative/technical/material support: Matschke. Study supervision: Aberle, Flitsch.

\section{Correspondence}

Amir-Hossein Rahvar: University Medical Center HamburgEppendorf, Hamburg, Germany. a.rahvar@uke.de. 